\title{
Primary melanoma of the penis
}

\section{Melanoma primario de pene}

Dorge Gerardo Sandoval-Téllez, ${ }^{1}$ iDAbel Antonio Ricardez-Espinosa, ${ }^{1}$

iDLuis Alfredo Jiménez-López, ${ }^{1}$ Edgar Pardo-Castillo. ${ }^{1}$

Keywords: Melanoma, Cancer, Penis.

\section{Abstract}

Clinical case: A 54-year-old man had an unremarkable past medical history and was evaluated due to lower urinary tract symptoms. He presented with phimosis and a hard lesion was palpated in the glans penis. A dorsal slit of the foreskin was performed, revealing a black, warty tumor of stony-hard consistency. Treatment was total penectomy. There was hepatic, splenic, and left inguinal lymph node tumor progression, as well as blastic lesions at the L4, T7, and T9 levels. Temozolomide was the initial palliative treatment, followed by interferon alpha-2b and zoledronic acid. The patient died 16 months after diagnosis

Relevance: Melanoma of the penis is an aggressive, rapidly progressing, malignant cutaneous neoplasia, with a high mortality rate, that derives from atypical melanocytic proliferation. It accounts for less than $1 \%$ of the cases of penile cancer and less than $0.2 \%$ of all melanomas. Approximately 200 cases have been reported in the literature.

Clinical implications: Treatment is surgical, through total penectomy, but there is no standardized management. The most reasonable treatment option appears to be radical surgery, when possible, together with adjuvant therapy.

Conclusions: Malignant melanoma of the penis is a cutaneous tumor of poor prognosis, given its great capacity to produce metastases, especially through lymphatic dissemination. Chemotherapy and radiotherapy are only palliative or adjuvant treatments and offer varying results.

Citación: Sandoval-Téllez J. G., Ricardez-Espinosa A. A., Jiménez-López, L. A., Pardo-Castillo, E. Melanoma primario de pene. Rev. Mex. Urol. 2019;79(6):pp 1-7.

Correpondencia: *Jorge Gerardo Sandoval-Téllez, Correo electrónico: drgerardosandoval@ gmail.com
${ }^{1}$ Instituto Mexicano del Seguro Social, Ciudad de México, México.

Recepción: 14 de mayo de 2019

Aceptación: 30 de diciembre de 2019

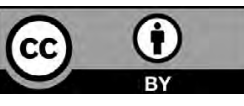




\section{Melanoma primario de pene. Sandoval-Téllez J. G., et al.}

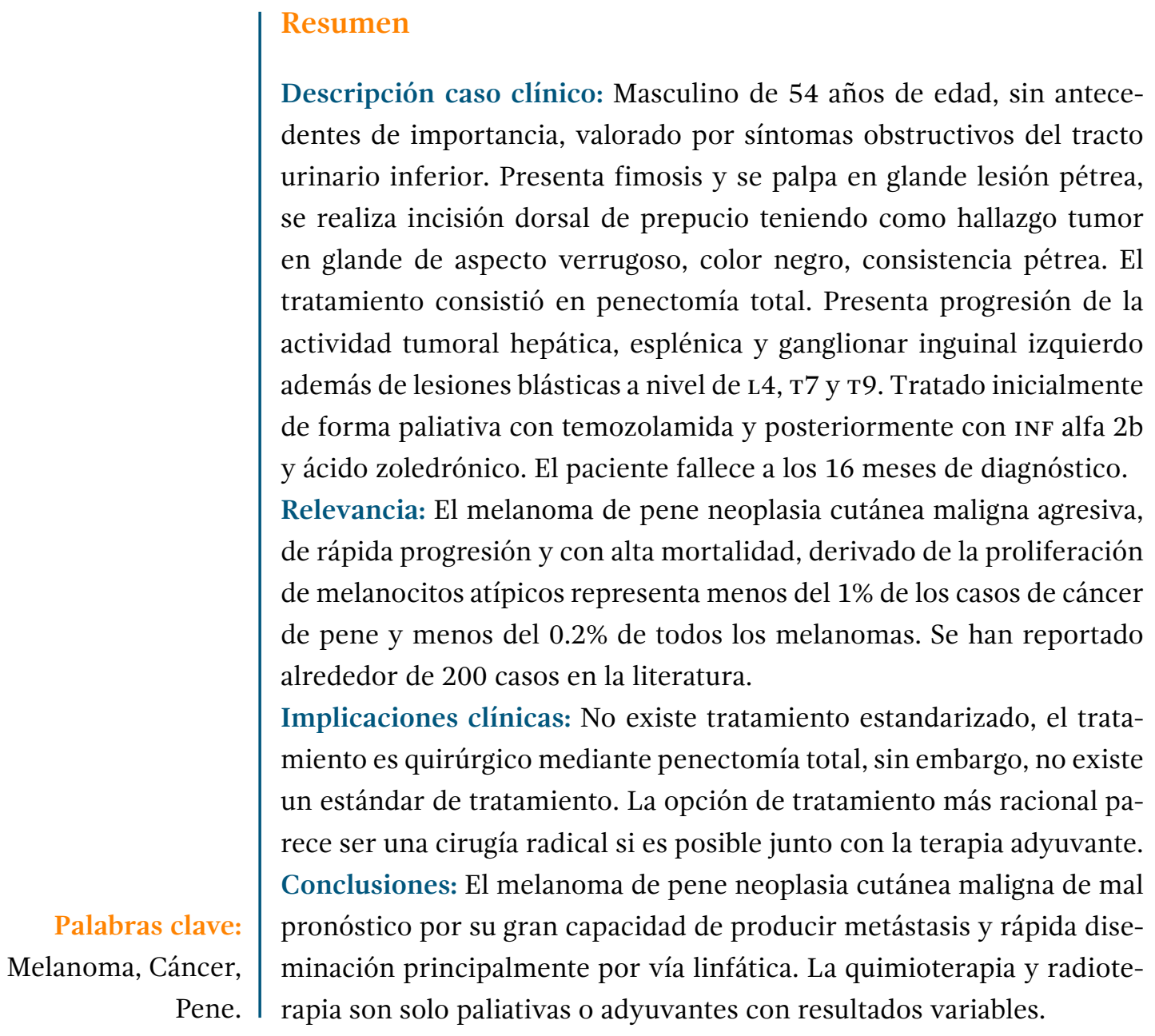

\section{Antecedentes}

El melanoma es un cáncer derivado de la proliferación de melanocitos atípicos, con o sin la capacidad de producir pigmento, caracterizado por su gran capacidad de metástasis. ${ }^{(1)}$ Constituye solamente entre el 1 y $4 \%$ de los cánceres de piel, sin embargo, es responsable de la mayoría de las muertes secundarias a esta neoplasia. $^{(2)}$

Además, representa menos del $1 \%$ de los casos de cáncer de pene y menos del $0.2 \%$ de todos los melanomas. ${ }^{(3-5)}$ Ocurre típicamente entre la sexta y séptima década de vida. ${ }^{(6)} \mathrm{Se}$ han reportado alrededor de 200 casos en la literatura desde el primer caso de melanoma de pene descrito por Muchnison en 1859. . $^{(7) 8)}$

\section{Caso clínico}

Paciente masculino de 54 años de edad. Originario del estado de Veracruz, sin antecedentes de importancia tanto heredofamiliares como 
personales patológicos para padecimiento actual, no padece enfermedades crónico-degenerativas y quien acudió a valoración por síntomas obstructivos del tracto urinario inferior.

Durante la exploración física a nivel de genitales se observa la presencia de fimosis y se palpa a nivel de glande lesión de consistencia pétrea, irregular e indolora. No se palpan adenopatías inguinales y no se observa alguna otra alteración en la exploración física restante.

Se procede a realizar incisión dorsal de prepucio teniendo como hallazgo tumor en glande de aspecto verrugoso, color negro, consistencia pétrea, abarcando hasta meato uretral (Fig. 1 y 2). Se decide tomar biopsia de la lesión y en un segundo tiempo quirúrgico se realiza penectomía total (Fig. 3), se propone realizar linfadenectomía, empero el paciente no acepta por las potenciales comorbilidades.

Figura 1. Tumor en glande, pigmentado, se aspecto irregular y de consistencia pétrea.

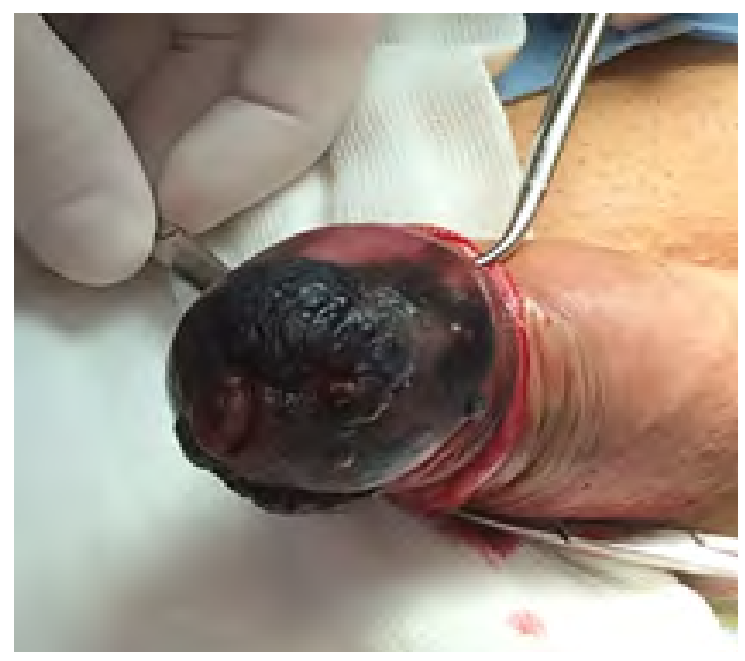

Figura 2. Tumor en surco balanoprepucial y áreas pigmentadas de forma difusa en glande.

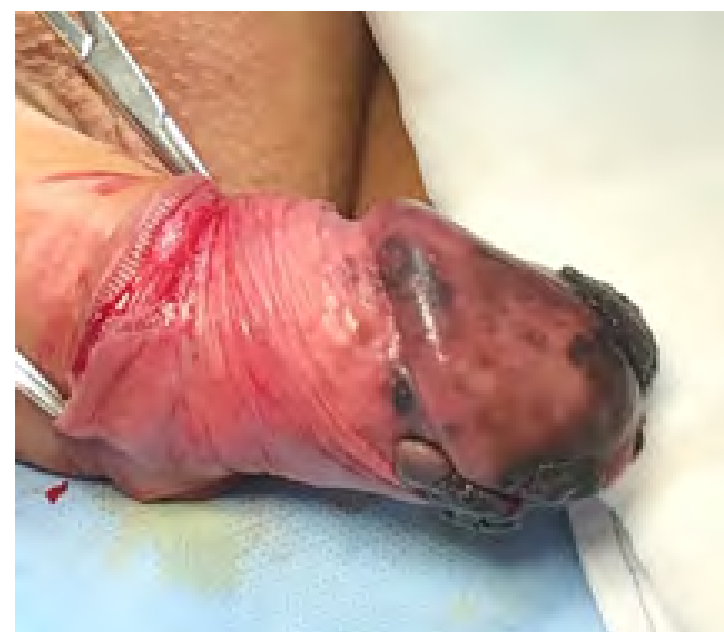

Figura 3. Penectomía total.

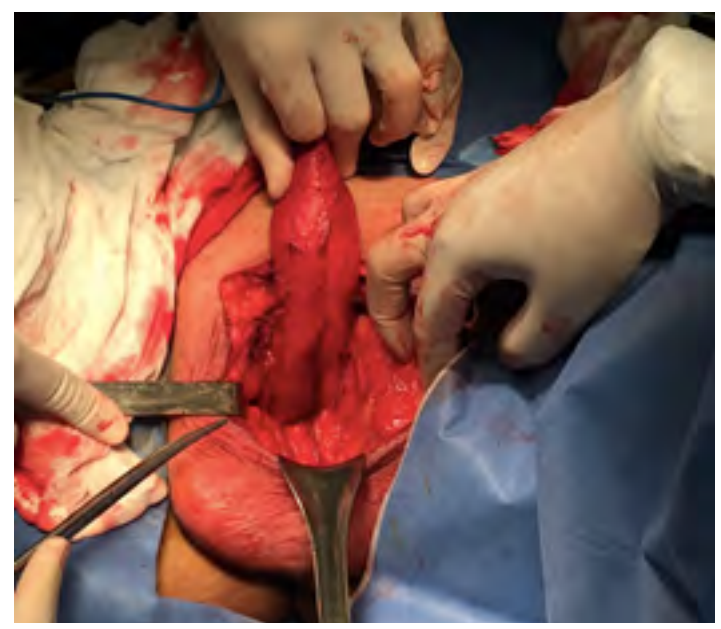

Reporte histopatológico: Melanoma nodular, multicéntrico, ulcerado, con infiltración a cuerpo esponjoso ( $2.5 \mathrm{~cm}$ de profundidad). Borde distal y marginal libre de lesión. Breslow 25 mm, 1 mitosis/mm2 (Fig. 4 y 5) 
Figura 4. Microfotografía 40x. Numerosas células melanocíticas atípicas con grandes núcleos hipercromáticos, eosinófilo y abundante citoplasma.

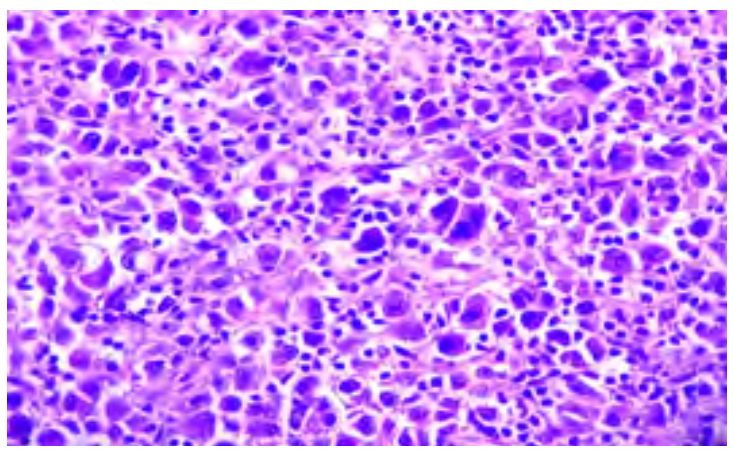

Figura 5. Microfotografía 4x. Neoplasia ulcerada. Melanocitos con disposición en nidos, con migración a epidermis característico de melanoma.

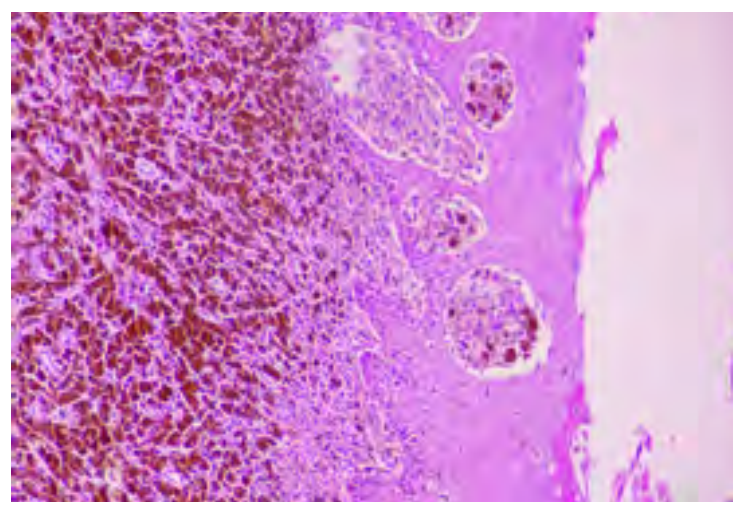

Dentro del seguimiento se observa aumento de volumen a nivel inguinal izquierdo a los 6 meses. Se realiza tomografía abdominopélvica, (Fig. 6) evidenciando patología a nivel inguinal izquierdo. Es valorado por cirugía oncológica ofreciendo lifandectomía con fines de estatificación. El paciente no acepta procedimiento quirúrgico por lo que se envía a oncología médica, iniciando tratamiento paliativo con temozolamida. Persiste con progresión de la actividad tumoral a nivel hepático, esplénico y ganglionar inguinal izquierdo. Nuevo estu- dio de tomografía evidencia progresión en el número y tamaño de las metástasis hepáticas y esplénicas, se identifican metástasis óseas; lesiones blásticas a nivel de L4, T7 y T9. Oncología medica cambia a tratamiento paliativo con INF alfa $2 b$ y ácido zoledrónico. Finalmente, el paciente fallece 16 meses después del diagnóstico secundario a falla hepática y falla renal.

Figura 6. Tomografía abdominopelvica. Se observan ganglios inguinales izquierdos de 39x37x34 mm. Densidades de 30 a 70 uh.

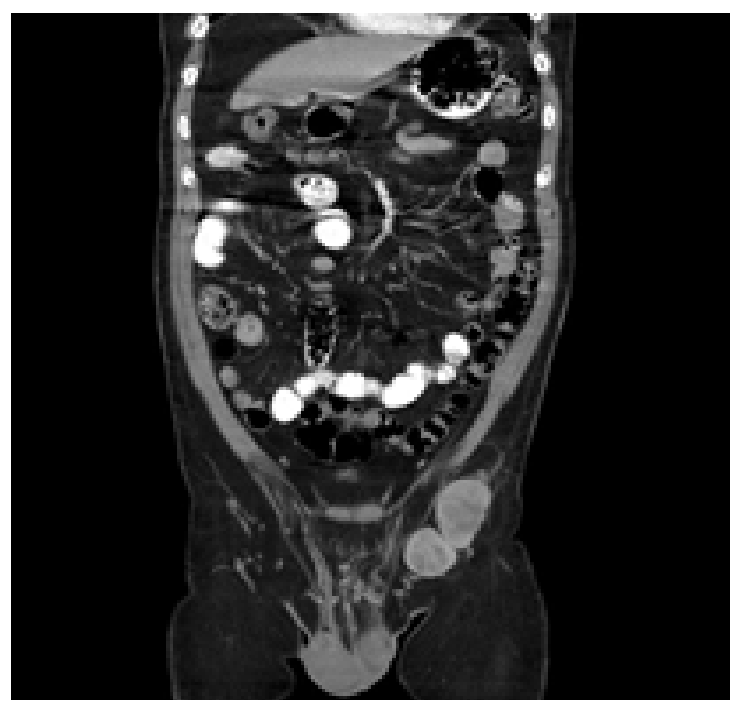

\section{Discusión}

El melanoma es una neoplasia cutánea maligna poco frecuente, pigmentada, plana o exofítica de mal pronóstico por su gran capacidad de producir metástasis y rápida diseminación principalmente por vía linfática, responsable de $75 \%$ de las muertes por cáncer de piel. ${ }^{(9)}$ Se calcula que la incidencia global del melanoma fue de 1.01/100 mil habitantes. ${ }^{(10)}$

El melanoma puede presentarse también como un tumor primario en otros órganos 
como tracto digestivo, respiratorio, sistema nervioso central, coroides, mucosas bucal o vaginal, independientemente de la localización de las metástasis. El melanoma realiza metástasis a la mayoría de los órganos con una especial predilección a ganglios, pulmón, hígado y cerebro.

(11) La mayoría de los melanomas se localizan en la piel representando el $95 \%$ de los casos y porcentaje restante en mucosa oral o genital, en retina y meninges. Se ha documentado que un $3 \%$ de pacientes desarrollan melanomas ocultos (enfermedad metastásica sin evidencia de tumor primario). ${ }^{(1)}$

A pesar de que el melanoma puede afectar a cualquier parte del cuerpo humano, la afectación del tracto genitourinario se observa en menos del $1 \%$ de los casos. ${ }^{(12)}$ Su presentación clínica varía desde máculas, pápulas o placas de tonalidad negra, azul marrón de consistencia firme, nodular hasta ulceradas y de gran tamaño. ${ }^{(13)}$ La mayoría se presentan en el glande (55\%), seguido del prepucio (28\%), cuerpo del pene $(9 \%)$ y meato uretral ( $8 \%){ }^{(14)}$

El melanoma es un tumor maligno de los melanocitos, células derivadas del neuroectodermo. Es un tumor de mal pronostico porque puede metastatizar a cualquier órgano. El melanoma de pene presenta metástasis tempranamente por vía linfática, sin embargo, las metástasis a hígado, pulmón, hueso, cerebro y otros sitios distantes corresponden a diseminación hematógena. ${ }^{(15)}$

Las tasas de supervivencia global a los 2 y 5 años son de $63 \%$ y $31 \%$, respectivamente. (16) Su etiología sigue siendo desconocida y el diagnóstico se realiza mediante biopsia de la lesión. Ante el examen microscópico se presenta asimetría, desprendimiento de células pigmentadas en la dermis, aumento atípico de las uniones intercelulares al igual que necrosis de los melanocitos. ${ }^{(17)}$ Se ha documentado que la ausencia de ulceración es proporcional al aumento de la tasa de supervivencia específica y la supervivencia libre de enfermedad a los 5 años. $^{(18)}$

La predicción del curso clínico del melanoma se basa principalmente en el grosor del tumor. La clasificación de Breslow se correlaciona estrechamente con las estadísticas de sobrevida. Los factores pronósticos adversos son espesor ( $\geq 3,5 \mathrm{~mm})$, ulceración y diámetro ( $\geq 15 \mathrm{~mm}){ }^{(17)}$ Sin embargo, la evaluación del grosor tumoral por sí sola no es suficiente; otros factores importantes en el pronóstico son la extensión del tumor de la participación de las estructuras locales y si hay evidencia clínica o histopatológica de metástasis en los ganglios linfáticos inguinales o pélvicos, lesiones delgadas (menores $1 \mathrm{~mm}$ ), pero de alto riesgo, se asocian con niveles de Clark IV o v, ulceración, índice mitótico alto (más de seis por campo de alto poder), invasión linfática o vascular y escasez de melanina en el tumor. ${ }^{(19)}$

Tradicionalmente, la estadificación de la enfermedad, la elección del tratamiento y el pronóstico del paciente se han basado en un sistema desarrollado por Bracken et al., ${ }^{(20)}$ con enfermedad en estadio I confinada al pene, estadio II metastásico a los ganglios linfáticos regionales y estadio III que representa diseminación enfermedad.

Hasta la fecha no existe tratamiento estandarizado. El tratamiento sigue siendo quirúrgico mediante penectomía. Anteriormente la regla era la penectomía total y linfadectomía ilioinguinal bilateral. ${ }^{(21)}$ Sin embargo, no existe un estándar de tratamiento, se ha reportado que la amputación distal con márgenes de excisión amplios puede ser suficiente, siendo controvertida hoy día la linfadenectomía pro- 
filáctica rutinaria en el melanoma peneano. ${ }^{(22)}$ La opción de tratamiento más racional parece ser una cirugía radical si es posible junto con la terapia adyuvante. ${ }^{(23)}$

La quimioterapia está indicada para el melanoma diseminado. La combinación quimioterapia consistente en seis ciclos de dacarbazina (DTIC), carmustina (вCNU), cisplatino y tamoxifeno presenta el mejor resultado. La tasa de respuesta a la terapia varía entre 15\% y $45 \%$. ${ }^{(22)}$ Se han descrito esquemas de quimioterapia con un solo agente agentes como dacarbazina, temozolomida o cisplatino. ${ }^{(24)}$ Se ha informado que la interleuquina- 2 y el interferón alfa- $2 b$ mejoran la supervivencia de pacientes seleccionados con buen estado funcional. Finalmente, la terapia blanco se ha centrado en inhibidores moleculares del gen BRAF y c-KIT. La inmunoterapia dirigida contra el factor de crecimiento endotelial vascular usando bevacizumab y los reguladores del sistema inmunitario CTLA-4 y PD-1. ${ }^{(25)}$

\section{Conclusión}

El melanoma de pene es una enfermedad agresiva, de rápida progresión y con alta mortalidad. El tratamiento consiste en la penectomía total. El papel de la linfadectomía profiláctica de forma rutinaria continúa siendo controversial. Los tratamientos de quimioterapia y radiación son solo paliativos o adyuvantes con resultados variables. Los pacientes con enfermedad metastásica tienen un mal pronóstico y no se ha demostrado que ningún medicamento en monoterapia o en combinación haya ejercido un impacto significativo en la supervivencia de los pacientes.

\section{Bibliografía}

1. Fuente-García A de la, Ocampo-Candiani J. Melanoma cutáneo. Gac Med Mex. 2010;146(2):126-35.

2. Sabatini-Ugarte $\mathbf{N}$, Molgó $\mathbf{M}$, Vial G. Melanoma en Chile ¿Cuál es nuestra realidad? Revista Médica Clínica Las Condes. 2018 Jul 1;29(4):468-76. doi: https://doi.org/10.1016/j. rmclc.2018.06.006

3. Iversen K, Robins RE. Mucosal malignant melanomas. The American Journal of Surgery. 1980 May 1;139(5):660-4. doi: https://doi. org/10.1016/0002-9610(80)90358-X

4. Stillwell Thomas J., Zincke Horst, Gaffey Thomas A., Woods John E. Malignant Melanoma of the Penis. Journal of Urology. $1988 \mathrm{Jul}$ 1;140(1):72-5. doi: https://doi.org/10.1016/ S0022-5347(17)41490-X

5. Cold CJ, Taylor JR. The prepuce. BJU International. 1999;83(S1):34-44. doi: https:// doi.org/10.1046/j.1464-410x.1999.0830s1034.x

6. Seetharamu N, Ott PA, Pavlick AC. Mucosal melanomas: a case-based review of the literature. Oncologist. 2010;15(7):772-81. doi: https:// doi.org/10.1634/theoncologist.2010-0067

7. Te CC, Vemulapalli S, Confer SD, Culkin DJ. Recurrent Malignant Melanoma of the Penis. Urology. 2008 Nov 1;72(5):1185.e151185.e16. doi: https://doi.org/10.1016/j. urology.2008.01.029

8. Gross S. A System of Surgery. 6th ed. Philadelphia: W.B.Saunders; 1882.

9. Herrera González NE, Flores AYA. Melanoma in Mexico. Rev Esp Med Quir. 2010;15(3):161-4.

10. Parada RJ, Pineda B, León G. Melanoma maligno cutáneo. Perfil epidemiológico en México. Gac Med Oncol. 2003;2(2):17-22.

11. Frías Ancona G, Hidalgo CO, Hernández MEL. Estudio epidemiológico de melanoma maligno 
Melanoma primario de pene. Sandoval-Téllez J. G., et al.

en el American British Cowdray Medical Center. Anales Médicos de la Asociación Médica del Centro Médico ABC. 2011;56(4):196-204.

12. Kim CJ, Pak K, Hamaguchi A, Ishida A, Arai Y, Konishi T, et al. Primary malignant melanoma of the female urethra. Cancer. 1993;71(2):44851. doi: https://doi.org/10.1002/10970142 ( 19930115 ) $71: 2 \% 3$ C 448 : : A I D CNCR2820710227\%3E3.0.CO;2-Y

13. Papeš D, Altarac S, Arslani N, Rajković Z, Antabak A, Ćaćić M. Melanoma of the glans penis and urethra. Urology. 2014;83(1):6-11. doi: https://doi.org/10.1016/j.urology.2013.07.009

14. Demitsu T, Nagato H, Nishimaki K, Okada O, Kubota T, Yoneda K, et al. Melanoma in situ of the penis. Journal of the American Academy of Dermatology. 2000 Feb 1;42(2, Part 2):386-8. doi: https://doi.org/10.1016/S01909622(00)90120-8

15. Turnbull N, Shim T, Patel N, Mazzon S, Bunker C. Primary Melanoma of the Penis in 3 Patients With Lichen Sclerosus. JAMA Dermatol. 2016 Feb 1;152(2):226-7. doi: http://dx.doi. org/10.1001/jamadermatol.2015.3404

16. van Geel AN, den Bakker MA, Kirkels W, Horenblas S, Kroon BBR, de Wilt JHW, et al. Prognosis of primary mucosal penile melanoma: a series of 19 Dutch patients and 47 patients from the literature. Urology. 2007 Jul;70(1):143-7. doi: https://doi.org/10.1016/j. urology.2007.03.039

17. Carli P, Giorgi VD, Soyer H, Stante $\mathbf{M}$, Mannone F, Giannotti B. Dermatoscopy in the diagnosis of pigmented skin lesions: a new semiology for the dermatologist. Journal of the European Academy of Dermatology and Venereology. 2000;14(5):353-69. doi: https:// doi.org/10.1046/j.1468-3083.2000.00122.x

18. Gajdos C, Griffith KA, Wong SL, Johnson TM, Chang AE, Cimmino VM, et al. Is there a benefit to sentinel lymph node biopsy in patients with T4 melanoma? Cancer. 2009;115(24):5752-60. doi: https://doi.org/10.1002/cncr.24660

19. Viveros EJM, Montor JGM, Moscoso IE, Ferro FC. Melanoma de pene. Presentación de un caso y revisión de la literatura. Rev Mex Urol. 2005;65(4):277-80.

20. Bracken RB, Diokno AC. Melanoma of the penis and the urethra: 2 case reports and review of the literature. J Urol. 1974 Feb;111(2):198200. doi: https://doi.org/10.1016/s00225347(17)59926-7

21. Sánchez-Ortiz Ricardo, Huang Samuel F., Tamboli Pheroze, Prieto Victor G., Hester Greg, Pettaway Curtis A. Melanoma of the penis, scrotum and male urethra: a 40-year single institution experience. Journal of Urology. 2005 Jun 1;173(6):1958-65. doi: https://doi. org/10.1097/01.ju.0000159207.91737.53

22. Bechara GR, Schwindt AB de S, Ornellas AA, Silva DEA da, Lott FM, Campos FS de, et al. Penile primary melanoma: analysis of 6 patients treated at Brazilian national cancer institute in the last eight years. International braz $\mathrm{j}$ urol. 2013 Dec;39(6):823-31. doi: http://dx.doi. org/10.1590/S1677-5538.IBJU.2013.06.08

23. Finn L, Markovic SN, Joseph RW. Therapy for metastatic melanoma: the past, present, and future. BMC Medicine. 2012 Mar 2;10(1):23. doi: https://doi.org/10.1186/1741-7015-10-23

24. Tsao H, Atkins MB, Sober AJ. Management of Cutaneous Melanoma. New England Journal of Medicine. 2004 Sep 2;351(10):998-1012. doi: https://doi.org/10.1056/NEJMra041245

25. Johnson Michael H., Brandes Steven B., Humphrey Peter A. Recurrent and Metastatic Malignant Melanoma of the Penis. Journal of Urology. 2012 Apr 1;187(4):1438-9. doi: https://doi.org/10.1016/j.juro.2012.01.024 